\title{
Electrical Brain Stimulation During a Retrieval-Based Learning Task Can Impair Long-Term Memory
}

\author{
Wesley Pyke ${ }^{1} \cdot$ Athanasios Vostanis $^{2} \cdot$ Amir-Homayoun Javadi $^{1,3,4}$ (I)
}

Received: 19 April 2020 / Accepted: 11 November 2020 / Published online: 26 November 2020

(C) The Author(s) 2020

\begin{abstract}
Anodal transcranial direct current stimulation (tDCS) to the left dorsolateral prefrontal cortex (DLPFC) has been shown to improve performance on a multitude of cognitive tasks. These are, however, often simple tasks, testing only one cognitive domain at a time. Therefore, the efficacy of brain stimulation for complex tasks has yet to be understood. Using a task designed to increase learning efficiency, this study investigates whether anodal tDCS over the left DLPFC can modulate both learning ability and subsequent long-term memory retention. Using a within-subject design, participants $(N=25)$ took part in 6 training sessions over consecutive days in which active or sham stimulation was administered randomly ( 3 of each). A computer-based task was used, containing flags from countries unknown to the participants. Each training session consisted of the repetition of 8 pairs of flag/country names. Subsequently, in three testing sessions, free, cued, and timed cued recall, participants were assessed on all 48 flags they had learnt. No difference in learning speed between active and sham tDCS was found. Furthermore, in the timed cued recall phase, flags learnt in the sham tDCS sessions were recalled significantly better than flags learnt in the active tDCS sessions. This effect was stronger in the second testing session. It was also found that for the flags answered incorrectly; thus, meaning they were presented more frequently, subsequent long-term retention was improved. These results suggest that for a complex task, anodal tDCS is ineffective at improving learning speed and potentially detrimental to long-term retention when employed during encoding. This serves to highlight the complex nature of brain stimulation, providing a greater understanding of its limitations and drawbacks.
\end{abstract}

Keywords Memory impairment · Long-term memory · Anodal stimulation · Transcranial direct current stimulation (tDCS) · Transcranial electrical brain stimulation (tES) $\cdot$ Retrieval-based learning $\cdot$ Dorsolateral prefrontal cortex (DLPFC)

\section{Introduction}

Cognitive enhancement using transcranial electrical brain stimulation has received much interest in the past decade, with a wide variety of methods showing improvements for memory (Javadi \& Walsh, 2012; Katz et al., 2017), reaction time (Hill, Fitzgerald, \& Hoy, 2016; Loftus, Yalcin, Baughman,

Amir-Homayoun Javadi

a.h.javadi@gmail.com

1 School of Psychology, Keynes College, University of Kent, Canterbury CT2 7NP, UK

2 The Tizard Centre, University of Kent, Canterbury, UK

3 Institute of Behavioural Neuroscience, University College London, London, UK

4 School of Rehabilitation, Tehran University of Medical Sciences, Tehran, Iran
Vanman, \& Hagger, 2015), and motor learning (Antal et al., 2004; Nitsche et al., 2003). The tasks utilised throughout these experiments have, however, targeted only one aspect of cognition (e.g. memorisation), which may have limited transferability to real life applications. Here, we investigated whether transcranial direct current stimulation (tDCS), previously shown to improve cognitive functioning (Coffman, Clark, \& Parasuraman, 2014), can be beneficial for a more complex task utilising retrieval-based learning.

A retrieval-based learning (RBL) task requires the participant to retrieve or re-access newly learnt material by undergoing tests. A traditional RBL task would consist of a study block (S), in which the participant is exposed to the target stimuli, then a testing block (T), where they are tested with no feedback. There can be different combinations of S and T (e.g. STST and SSTST). This has been shown to be more effective for learning than just exposure (SSSS) alone (Agarwal, Karpicke, Kang, Roediger, \& McDermott, 2008; 
Fazio \& Marsh, 2019; Karpicke \& Grimaldi, 2012; Karpicke, Lehman, \& Aue, 2014; Karpicke \& Roediger, 2007, 2008; Roediger \& Butler, 2011; Roediger \& Karpicke, 2006) (for a review, see Karpicke \& Aue, 2015). One reason for this is said to be due to a reconsolidation effect, where the memory trace is elaborated and alternative pathways are made, thus, creating an overall stronger and more accessible memory (McDaniel \& Masson, 1985; Roediger \& Butler, 2011; Sara, 2000).

The task used in this study is a variation of a SAFMEDS task, an acronym for Say All Fast a Minute Every Day Shuffled. Developed and used by applied behavioural analysts (ABA) in the late 1970s (Graf \& Auman, 2005), a SAFMEDS task utilises an operant conditioning paradigm, with learning facilitated through retrieval, repetition, and positive reinforcement. Whilst the SAFMEDS task has never previously been compared to a retrieval-based learning task, there are many similarities. In a retrieval/feedback loop, participants are constantly tested, with an emphasis on both speed and accuracy. Instead of having an ending criterion with a set number of trials, as per previous cognitive tasks, it employs a performance-based ending threshold. Each block is separated into 1-min timings, where exposure to stimuli is variable. Eventually, participants must provide a set number of correct responses in $1 \mathrm{~min}$ to complete the task. Invariably, this task will require substantial executive functioning across multiple cognitive domains. Previous research into retrieval has assessed working memory (WM; Rosen \& Engle, 1997) and long-term memory (LTM; Karpicke \& Roediger, 2007; Roediger \& Karpicke, 2006). By employing this type of learning, the variability in encoding techniques can be reduced, thus allowing for a clearer understanding of how particular interventions can modulate cognition.

There is much literature in support of transcranial direct current stimulation (tDCS) as a method of enhancement across multiple cognitive domains, dependent on the task and the site of stimulation (Bestmann, de Berker, \& Bonaiuto, 2015; Coffman et al., 2014; Parkin, Ekhtiari, \& Walsh, 2015). However, tDCS is not without its critics, with several studies showing null (de Lara, Knechtges, Paulus, \& Antal, 2017) or even impairing (Brunyé, Smith, Horner, \& Thomas, 2018) effects on various aspects of cognition (for a review see Horvath, Forte, \& Carter, 2015a, 2015b). tDCS delivers a weak electrical current mostly at the site of the electrode placement. Theories suggest that tDCS has the potential to either induce depolarisation (anodal stimulation) or hyperpolarisation (cathodal stimulation) of neurons (Javadi, Brunec, Walsh, Penny, \& Spiers, 2014; Miniussi, Harris, \& Ruzzoli, 2013), which, in turn, can alter cortical activity causing variations in perception, cognition, and behaviour (Fertonani \& Miniussi, 2017; M. Nitsche et al., 2008).

Here, we apply tDCS concurrently with a type of retrievalbased learning task, to ascertain whether learning speed can be further accelerated, and whether the combination of the behavioural task and tDCS modulates retention of the LTM. The computerised task aimed to teach participants flags of the world unknown to them. Both free and cued recall ability were assessed in the test sessions. With previous literature highlighting the beneficial effects of tDCS, it was hypothesised that in the training sessions, active tDCS accelerates learning speed. As improvements have also been shown for LTM following tDCS, it was also hypothesised that subsequent long-term retention would also be improved for active tDCS relative to the sham $\mathrm{tDCS}$.

\section{Methods}

\section{Participants}

A total of 25 participants took part in the experiment (21 females, 4 males, age range $18-21$, mean $[\mathrm{SD}]=19.20$ [0.84]). All participants spoke fluent English and had normal or corrected to normal vision and hearing, and did not report any neurological or learning difficulties.

\section{Study Design}

The study adopted a within-subject design, with an initial baseline measure completed in the first session to identify how many flags the participant already knew. Participants who knew more than 12 flags (out of 60) were excluded from the study. See Appendix for the list of the countries. Following this, over the course of six 30-min training sessions, participants learnt 48 flags ( 8 flags per session). These six sessions were split over three active and three sham tDCS, which were pseudo-randomly assigned to each training session with neither stimulation type repeated three times in a row. See Fig. 1a for a demonstration of the procedure.

\section{Behavioural Task}

Following set up and initiation of the brain stimulation, training sessions consisted of a computerised variation of a SAFMEDS task, with 8 different flags of uncommon countries presented in each session. Flags were chosen as stimuli as these were deemed to provide more motivation to learn than, for example, abstract random symbols. Whilst it should be acknowledged that flags may possess similarities or elements of uniqueness that may cause differences in the ability to remember them, the authors believed that the motivation to learn the flags was a more important factor. Previous research has shown that motivation is the key to successful learning (Michael, 1993; Olson, Laraway, \& Austin, 2001; Sundberg, 1993; Zayac \& Johnston, 2008). The aim was for participants to learn all 8 flags shown in the session and be able to repeat them 60 times in $1 \mathrm{~min}$ (recommended frequency aims for 
(a)
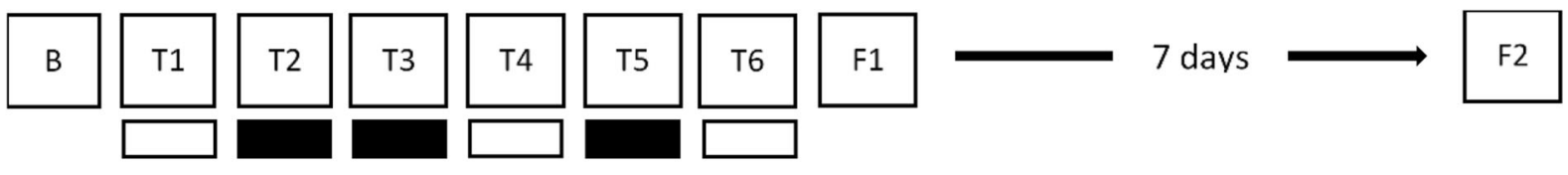

(b)

block 1

block 2

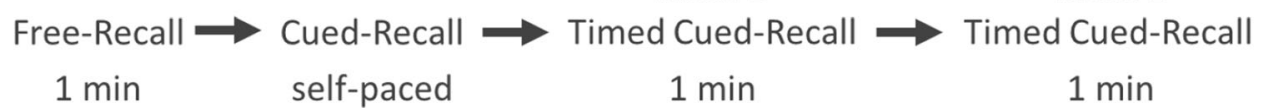

Fig. 1 Procedure of the study. a Visual timeline of all the sessions. B indicates the baseline session, T1-T6 indicate the six 30-min training sessions. F1 and F2 indicate the two 30-min testing (follow-up) sessions, separated by 7 days. All sessions (apart from F2) were conducted on

consecutive days. Black and white boxes give an example of how the randomisation pattern for the stimulation may have looked; black, active tDCS; white, sham tDCS. No stimulation was administered in any other sessions. b Procedure of the testing sessions (follow-ups 1 and 2)

'See/Say task'; Fabrizio \& Moors, 2017). Training was split over 1-minute blocks with flags displayed one at the time. See Fig. 2 for the procedure of each training trial. Following onset of the flag, participants were asked to say the name of the country, or say 'go' indicating that they do not know or remember the name. If the participant took longer than $3 \mathrm{~s}$ to respond, they were encouraged to say ' $g o$ ' and continue to the next flag. This would be recorded as an incorrect response. Following the participant's response, the experimenter indicated accuracy via a mouse-button click. To assist with pronunciation, for the first block, a computerised voice read aloud the name of the country upon mouse click by the researcher, before moving on to the next flag. After the participant had correctly answered each flag four consecutive times, the experimenter no longer had to indicate a correct or incorrect answer (it was assumed to be correct) and no further feedback was provided. This gave full control of pace to the participant, allowing them to cycle through the flags at speed. Accuracy of the responses were monitored constantly. If an incorrect answer was given at any point during this stage, the experimenter would interrupt the participant with the correct answer.
Training continued until the participant could reach the threshold of 60 flags per minute or a maximum of 17 blocks. Whilst participants were aware of the aim of the study, they were told not to practise the task outside of the sessions.

\section{Testing Sessions (Follow-ups 1 and 2)}

See Fig. $1 \mathrm{~b}$ for the procedure of the testing session. Testing sessions consisted of three phases testing different aspects of participants' memory performance: free recall, cued recall, and cued recall under timed pressure. In the first phase, participants were asked to recall as many of the countries of the flags they had learnt during the six training sessions ('free recall' phase). This was timed for $1 \mathrm{~min}$ and their responses were recorded on a voice recorder.

In the second phase, all the 48 flags were presented in random order and participants were asked to recall the name of the countries without any time pressure ('cued recall' phase). Auditory and visual feedback was provided following the participant's response. Feedback was provided to refresh participant's memory as well as providing them with an

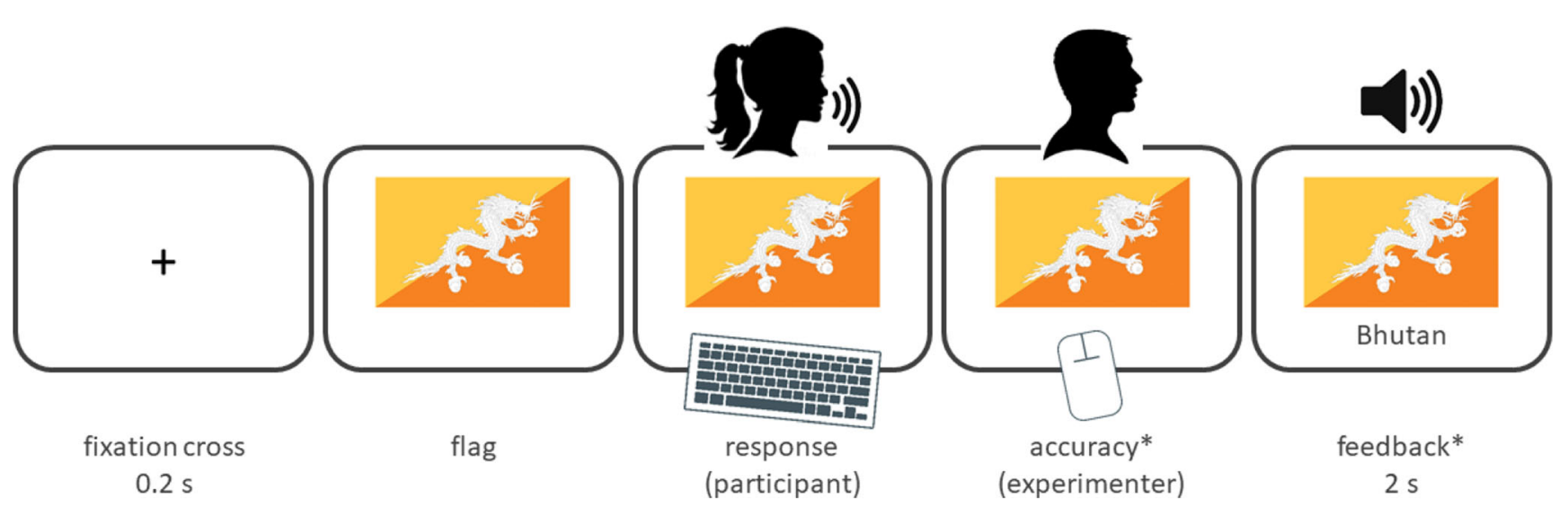

Fig. 2 Procedure of each trial in the training sessions (T1-T6). Participant's task was to say aloud the name of the country, or 'go' to indicate that they do not know the name. Following that they were instructed to press the space key. Following participant's response, the experimenter indicated the accuracy of the response using mouse clicks.
*The experimenter was no longer required to indicate accuracy of the response and no feedback was given, following four consecutive correct responses on each flag. Auditory feedback was given only in the 1st block of each training day 
opportunity to see all of the flags in one session. This also prepared them for the third and final phase in which their ability under time pressure was assessed.

Flags presented in the training sessions were grouped into two blocks (24 flags in each block), based on the stimulation condition of the training session they were presented in one block for all the flags presented in sham tDCS training sessions and one block for the rest of the flags. These two blocks were presented in random order in the final phase of the testing session. In this phase, participants took part in a timed cued recall task consisting of two 1-min blocks ('timed cued recall' phase). No feedback was provided in this phase. Correct and incorrect answers were marked by the researcher on paper, on a list generated prior to the study. The two testing sessions followed the same procedure.

\section{Transcranial Direct Current Stimulation}

We applied tDCS over the left dorsolateral prefrontal cortex (left DLPFC), as this has been shown to be involved in a number of cognitive processes, including decision-making performance (Hecht, Walsh, \& Lavidor, 2010; Philiastides, Auksztulewicz, Heekeren, \& Blankenburg, 2011), WM performance (Andrews, Hoy, Enticott, Daskalakis, \& Fitzgerald, 2011; Fregni et al., 2005; Zaehle, Sandmann, Thorne, Jäncke, \& Herrmann, 2011) (for a review, see (Brunoni \& Vanderhasselt, 2014)), reaction time (RT) (Brunoni \& Vanderhasselt, 2014; Loftus et al., 2015; Zaehle et al., 2011), and LTM (Dedoncker, Brunoni, Baeken, \& Vanderhasselt, 2016; Gray, Brookshire, Casasanto, \& Gallo, 2015; Javadi \& Cheng, 2013; Javadi \& Walsh, 2012; Leshikar et al., 2017; Manenti, Brambilla, Petesi, Ferrari, \& Cotelli, 2013). One anodal $5 \times 7 \mathrm{~cm}^{2}$ saline-soaked surface sponge was placed over the left DLPFC (F3, 10-20 international method for measurement (Homan, Herman, \& Purdy, 1987)) and held in place using a bandage and clips. The reference electrode was placed on the top of the participant's left wrist. An extracephalic site was used to avoid any possible cathodal stimulation of the reference site. Additionally, it was deemed to be the least invasive of other common extracephalic sites such as the shoulder (previous studies using wrist; Accornero et al., 2014; Fertonani \& Miniussi, 2017; Nasseri, Nitsche, \& Ekhtiari, 2015). See Fig. 3 for the modelling of the current flow using this montage. The stimulation device used was a DC Brain Stimulator Plus (NeuroConn, Ilmenau, Germany). For the active tDCS conditions, $1.5 \mathrm{~mA}$ of anodal stimulation with 10-s fade-in and fade-out was delivered for $15 \mathrm{~min}$. The study adopted a mixed stimulation protocol, with stimulation starting $5 \mathrm{~min}$ prior to task onset and continuing for $10 \mathrm{~min}$ during the task. In the active tDCS condition, stimulation started 5 min prior to the beginning of the task to allow the stimulation to take effect and to allow the participant to familiarise themselves with the sensation accompanying the

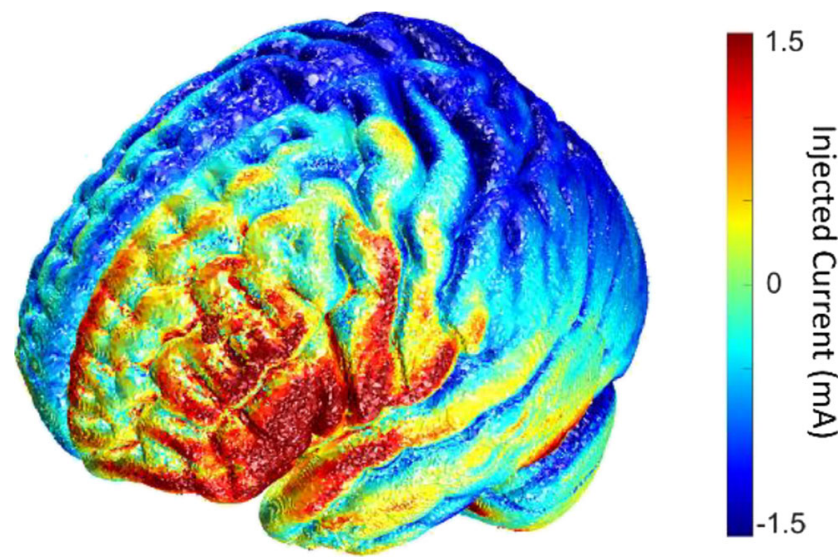

Fig. 3 Modelling of the current flow showing concentration of the stimulation over the target area, the left dorsolateral prefrontal cortex (left DLFPC). Anode electrode was placed over left DLFPC (F3) and the cathode electrode was left on the left wrist. Both electrodes were $5 \times 7 \mathrm{~cm}^{2}$. Modelling was done using open-source software ROAST (Huang, Datta, Bikson, \& Parra, 2019)

stimulation. Although still up for debate, stimulating before task execution ('offline') has been previously shown to be as effective as online stimulation (Hill et al., 2016), with the time course lasting for more than an hour after cessation of stimulation (Manenti et al., 2015; Marangolo et al., 2013; Nitsche \& Paulus, 2000; for a review, see Au, Karsten, Buschkuehl, \& Jaeggi, 2017). For the sham tDCS condition, the procedure was identical, except stimulation was discreetly turned off after $20 \mathrm{~s}$. All participants believed they were receiving brain stimulation in some form and were told that varying protocols of stimulation were used, to account for differences in sensation at the site of stimulation.

\section{Statistical Analysis}

Data analyses were performed using SPSS (v25; LEAD Technologies, Inc., Charlotte, NC). Number of required training blocks for different stimulation conditions was analysed using a generalised estimating equation (GEE) with session and stimulation condition as within-subject factors. To further analyse the strength of support for the null hypothesis of enhanced performance in the active tDCS condition, Bayesian analysis was conducted. Number of times each flag was responded incorrectly ('total error') and the total number of presentations of each flag ('total exposure') was recorded for the analysis of the data in the testing sessions.

The data for the three phases of the two testing sessions (follow-ups 1 and 2) were analysed: number of flags remembered in the free recall phase, percentage of accurate recognition of the flags in the cued recall phase, and the speed of recognition of the flags in the timed cued recall phase. To investigate the effect of the two brain stimulation conditions, the recalled flags in the free recall phase were split into flags that were trained in active and sham tDCS sessions. Three 
GEE analyses were conducted with stimulation condition (active/sham tDCS) and session (follow-ups 1 and 2) as withinsubject factors. To further analyse the effect of total error and total exposure on memory performance, GEE analyses were run with these parameters as covariates. Finally, six post hoc GEE analyses were run to investigate the effect of brain stimulation on performance across the two testing sessions. Bonferroni correction is used to correct for multiple comparisons.

\section{Results}

\section{Training Phase}

Interobserver agreement data was not collected during the study, as it was not possible with the resources available. However, two steps were taken to ensure consistency in the way the training was delivered and how the participants' responses were scored. Firstly, we conducted a preliminary pilot study during which the first author received training in the process of the data collection and the use of the SAFMEDS software. A Board Certified Behaviour Analyst (BCBA), with over 6 years of experience, was responsible for overseeing the procedure. Second, after the completion of the study, we conducted a post hoc assessment of an interobserver agreement. Specifically, three additional participants, meeting the same criteria of inclusion, were recruited. Participants completed 10 training blocks each, across 2 days. The first author scored their responses, and the BCBA scored and calculated interobserver agreement. The percentage of agreement was $98 \%$.

A generalised estimating equation (GEE) was used to investigate the effect of brain stimulation on the number of required training blocks across the six training sessions. This analysis showed a significant main effect of session $(\beta=-$ $0.573, \mathrm{SE}=0.202,95 \% \mathrm{CI}=[-0.969,-0.177]$, Wald $\chi^{2}=$ $8.036, p=0.005$ ) showing a reduction in the number of training blocks with the progression of training sessions (session 1: mean $[\mathrm{SD}]=10.64$ [4.44], session 2: 9.44 [4.23], session 3: 9.20 [4.45], session 4: 8.56 [4.88], session 5: 7.68 [4.57], session 6: 7.92 [4.91]). The main effect of stimulation condition was not significant (sham tDCS 8.69 [4.28], active $\mathrm{tDCS}=9.12[3.89] ; \beta=0.229, \mathrm{SE}=1.311,95 \% \mathrm{CI}=[-$ $2.341,2.800]$, Wald $\left.\chi^{2}=0.031, p=0.861\right)$, nor the interaction effect $(\beta=0.037, \mathrm{SE}=0.344,95 \% \mathrm{CI}=[-0.637,0.712]$, Wald $\left.\chi^{2}=0.012, p=0.914\right)$. Bayesian analysis was conducted to investigate the strength of support for the null hypothesis. This analysis showed a high Bayes factor value $\left(\mathrm{BF}_{01}=\right.$ 4.827), which provides substantial evidence that brain stimulation had no effect on learning ability (Jarosz \& Wiley, 2014). See Fig. 4 for the distribution of the number of training blocks across the training sessions. Participants finished the training in less than 17 training blocks in majority of the training sessions (94\% of all training sessions).

\section{Free Recall}

The three phases of the two testing sessions were analysed separately. See Fig. 5 for the distribution of responses for the two stimulation conditions across the three testing phases. A GEE analysis on the number of recalled flags in the free recall phase showed a significant effect of session $(p=0.012)$ showing higher memory performance in the second testing session (follow-up 1: 20.67 [8.10]\%, follow-up 2: 29.01 $[9.43] \%)$. GEE analyses with total error and total exposure as covariates showed a significant effect of the two variables (total error: $p=0.031$, total exposure: $p=0.041$ ) indicating that the higher these variables, the more likely the flag is remembered correctly. See Table 1 for the details of these analyses.

\section{Cued Recall}

A GEE analysis on the number of recognised flags in the cued recall phase showed a significant effect of session $(p<0.001)$, showing higher memory performance in the second testing session (follow-up 1: 31.92 [13.90]\%, follow-up 2: 57.08 $[18.27] \%)$. GEE analyses with total error and total exposure as covariates showed significant effect of the two variables (total error: $p<0.001$, total exposure: $p=0.001$ ) indicating
Fig. 4 Number of training blocks required for each participant in each training session. Training was terminated after 17 training blocks to avoid exhaustion. The solid lines indicate the mean value

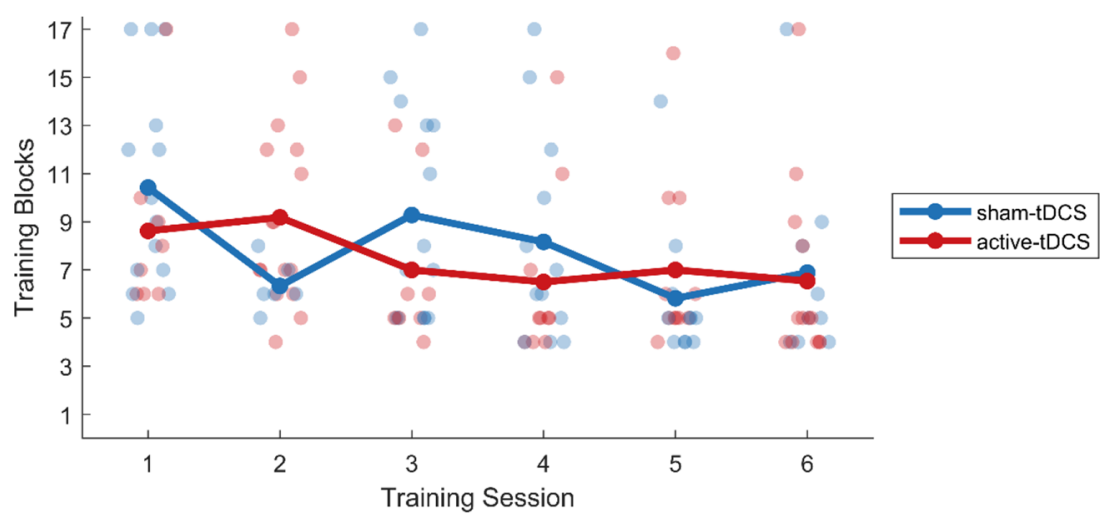




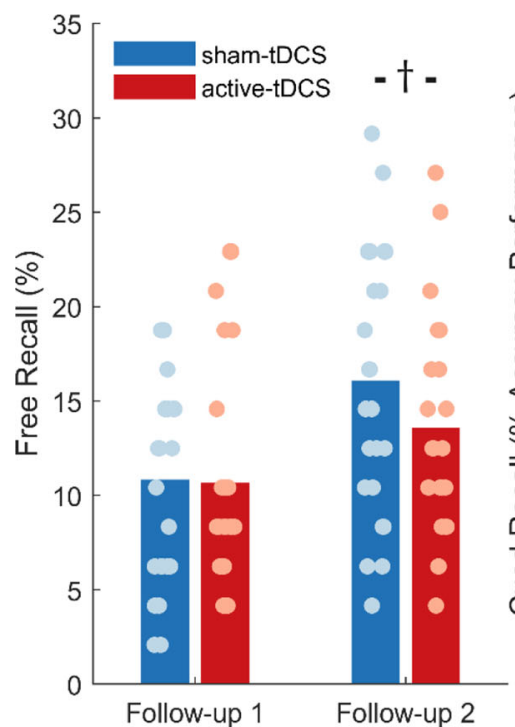

(a)

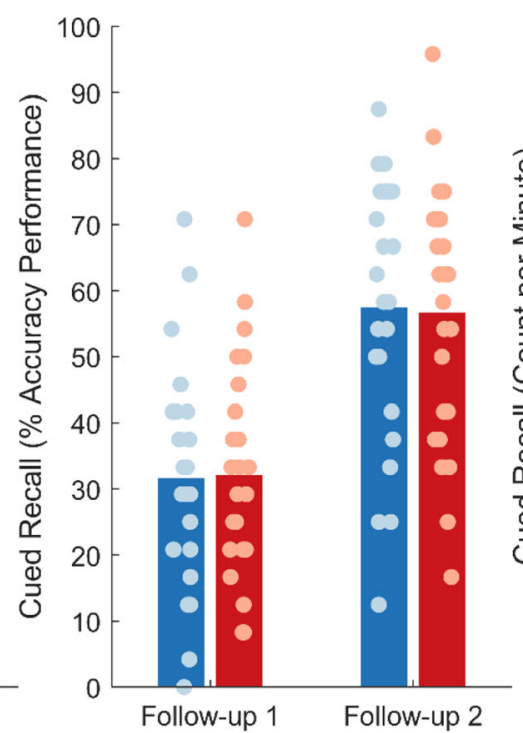

(b)

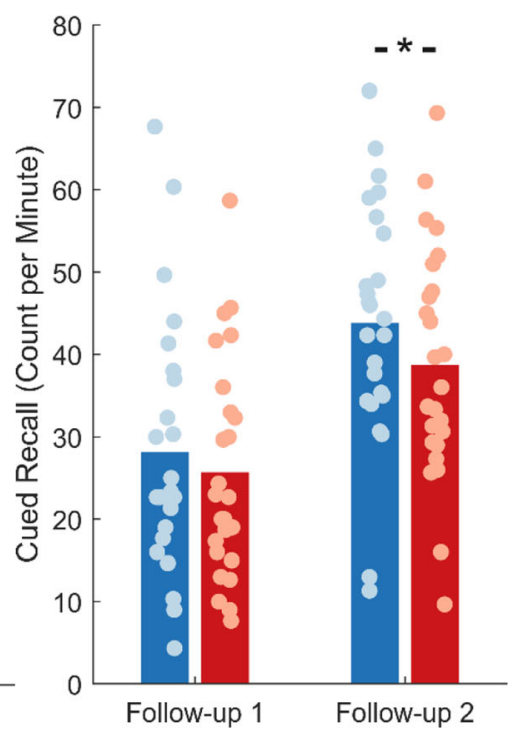

(c)

Fig. 5 Plots showing performance in the a free recall, $\mathbf{b}$ cued recall (self-paced), and $\mathbf{c}$ timed cued recall phases split over stimulation conditions (active/ sham tDCS) and sessions (follow-up 1 and 2). ${ }^{*} p<0.008$ Bonferroni corrected for multiple comparison, ${ }^{\dagger} p=0.141$ non-significant

that the higher these variables, the more likely the flag is remembered correctly. See Table 2 for the details of these analyses. The interaction of session and total exposure was also significant $(p<0.001)$. Therefore, we ran a GEE analysis on each session separately. These analyses showed a significant effect of total exposure in both sessions (follow-up 1: $\beta=$ $0.010, \mathrm{SE}=0.004,95 \% \mathrm{CI}=[0.002,0.018]$, Wald $\chi^{2}=5.416$, $p=0.020$, odds ratio $=1.010 ;$ Follow-up $2: \beta=0.019, \mathrm{SE}=$
Table 1 Summary of the generalised estimating equation (GEE) analyses with session and stimulation conditions as withinsubject factor and total error and total exposure as covariates in the free recall phase of the two testing sessions

\begin{tabular}{|c|c|c|c|c|c|c|}
\hline & $\beta$ & SE & $95 \% \mathrm{CI}$ & $\begin{array}{l}\text { Wald } \\
\chi^{2}\end{array}$ & $p$ & OR \\
\hline \multicolumn{7}{|l|}{ No covariate } \\
\hline Session & 0.332 & 0.133 & {$[0.072,0.592]$} & 6.252 & $0.012 *$ & 1.394 \\
\hline Stimulation condition & -0.211 & 0.143 & {$[-0.491,0.070]$} & 2.172 & 0.141 & 0.810 \\
\hline Session $\times$ stimulation condition & 0.231 & 0.209 & {$[-0.179,0.641]$} & 1.218 & 0.270 & 1.260 \\
\hline \multicolumn{7}{|l|}{ Total error } \\
\hline Session & 0.163 & 0.241 & {$[-0.309,0.635]$} & 0.457 & 0.499 & 1.177 \\
\hline Stimulation condition & -0.166 & 0.230 & {$[-0.616,0.284]$} & 0.522 & 0.470 & 0.847 \\
\hline Total error & 0.118 & 0.055 & {$[0.011,0.226]$} & 4.639 & $0.031 *$ & 1.125 \\
\hline Session $\times$ stimulation condition & 0.269 & 0.328 & {$[-0.375,0.912]$} & 0.670 & 0.413 & 1.308 \\
\hline Session $\times$ total error & 0.060 & 0.068 & {$[-0.074,0.193]$} & 0.770 & 0.380 & 1.061 \\
\hline Stimulation condition $\times$ total error & -0.011 & 0.074 & {$[-0.155,0.134]$} & 0.021 & 0.885 & 0.989 \\
\hline Three-way interaction & -0.011 & 0.086 & {$[-0.180,0.158]$} & 0.016 & 0.900 & 0.989 \\
\hline \multicolumn{7}{|l|}{ Total exposure } \\
\hline Session & 0.383 & 0.237 & {$[-0.081,0.847]$} & 2.616 & 0.106 & 1.467 \\
\hline Stimulation condition & -0.445 & 0.225 & $\begin{array}{l}{[-0.886} \\
\quad-0.005]\end{array}$ & 3.921 & $0.048 *$ & 0.641 \\
\hline Total exposure & 0.008 & 0.004 & {$[0.000,0.016]$} & 4.171 & $0.041 *$ & 1.008 \\
\hline Session $\times$ stimulation condition & 0.082 & 0.385 & {$[-0.672,0.836]$} & 0.046 & 0.831 & 1.086 \\
\hline Session $\times$ total exposure & -0.001 & 0.005 & {$[-0.011,0.009]$} & 0.065 & 0.799 & 0.999 \\
\hline $\begin{array}{l}\text { Stimulation condition } \times \text { total } \\
\text { exposure }\end{array}$ & 0.007 & 0.006 & {$[-0.004,0.018]$} & 1.592 & 0.207 & 1.007 \\
\hline Three-way interaction & 0.004 & 0.009 & {$[-0.012,0.021]$} & 0.265 & 0.607 & 1.004 \\
\hline
\end{tabular}

$O R$, odd ratio; $S E$, standard error 
Table 2 Summary of the generalised estimating equation (GEE) analyses with session and stimulation conditions as withinsubject factor and total error and total exposure as covariates in the cued recall phase of the two testing sessions

\begin{tabular}{|c|c|c|c|c|c|c|}
\hline & $\beta$ & $\mathrm{SE}$ & $95 \% \mathrm{CI}$ & $\begin{array}{l}\text { Wald } \\
\chi^{2}\end{array}$ & $p$ & OR \\
\hline \multicolumn{7}{|l|}{ No covariate } \\
\hline Session & 1.014 & 0.129 & {$[0.761,1.267]$} & 61.703 & $<0.001 *$ & 2.758 \\
\hline Stimulation condition & -0.034 & 0.091 & {$[-0.213,0.145]$} & 0.139 & 0.709 & 0.967 \\
\hline Session $\times$ stimulation condition & 0.057 & 0.144 & {$[-0.225,0.339]$} & 0.157 & 0.692 & 1.059 \\
\hline \multicolumn{7}{|l|}{ Total error } \\
\hline Session & 0.989 & 0.204 & {$[0.588,1.390]$} & 23.420 & $<0.001 *$ & 2.689 \\
\hline Stimulation condition & 0.186 & 0.218 & {$[-0.243,0.615]$} & 0.722 & 0.395 & 1.204 \\
\hline Total error & 0.348 & 0.055 & {$[0.239,0.457]$} & 39.150 & $<0.001 *$ & 1.416 \\
\hline Session $\times$ stimulation condition & 0.133 & 0.222 & {$[-0.302,0.568]$} & 0.360 & 0.549 & 1.142 \\
\hline Session $\times$ total error & 0.065 & 0.071 & {$[-0.075,0.206]$} & 0.832 & 0.362 & 1.067 \\
\hline Stimulation condition $\times$ total error & -0.057 & 0.067 & {$[-0.190,0.075]$} & 0.717 & 0.397 & 0.944 \\
\hline Three-way interaction & -0.044 & 0.091 & {$[-0.222,0.135]$} & 0.230 & 0.631 & 0.957 \\
\hline \multicolumn{7}{|l|}{ Total exposure } \\
\hline Session & 1.589 & 0.182 & {$[1.232,1.946]$} & 76.124 & $<0.001 *$ & 4.899 \\
\hline Stimulation condition & -0.327 & 0.233 & {$[-0.785,130]$} & 1.967 & 0.161 & 0.721 \\
\hline Total exposure & 0.015 & 0.004 & {$[0.007,0.024]$} & 11.919 & $0.001 *$ & 1.015 \\
\hline Session $\times$ stimulation condition & -0.372 & 0.260 & {$[-0.883,0.138]$} & 2.044 & 0.153 & 0.689 \\
\hline Session $\times$ total exposure & -0.014 & 0.004 & $\begin{array}{l}{[-0.022} \\
\quad-0.006]\end{array}$ & 12.285 & $<0.001 *$ & 0.986 \\
\hline $\begin{array}{l}\text { Stimulation condition } \times \text { total } \\
\text { exposure }\end{array}$ & 0.008 & 0.005 & {$[-0.002,0.019]$} & 2.734 & 0.098 & 1.009 \\
\hline Three-way interaction & 0.011 & 0.007 & {$[-0.004,0.027]$} & 2.166 & 0.141 & 1.011 \\
\hline
\end{tabular}

$O R$, odd ratio; $S E$, standard error
$0.004,95 \% \mathrm{CI}=[0.011,0.027]$, Wald $\chi^{2}=20.642, p<0.001$, odds ratio $=1.019$ ) with the effect slightly stronger in testing session follow-up 2 .

\section{Timed Cued Recall}

A GEE analysis of the timed cued recall showed a significant effect of session $(\beta=15.613, \mathrm{SE}=1.993,95 \% \mathrm{CI}=[11.707$, $19.520]$, Wald $\left.\chi^{2}=61.372, p<0.001\right)$, and no significant effect of stimulation condition $(\beta=-2.493, \mathrm{SE}=1.974,95 \%$ $\mathrm{CI}=[-6.362,1.375]$, Wald $\left.\chi^{2}=1.595, p=0.207\right)$, nor interaction of session and stimulation condition $(\beta=-2.587, \mathrm{SE}=$ $2.276,95 \% \mathrm{CI}=[-7.047,1.873]$, Wald $\chi^{2}=1.292, p=$ $0.256)$.

Although there was no significant interaction effect of stimulation condition and session, based on our strong hypothesis that stimulation improves performance, post hoc paired-sample GEE analyses were run to further investigate the differences between stimulation conditions over the two testing sessions (see Table 3 ). Timed cued recall was significantly $(p<0.001)$ better in the sham tDCS condition compared to active tDCS in the second testing session (followup 2; sham $\mathrm{tDCS}=43.81$ [14.66], active $\mathrm{tDCS}=38.73$ [14.14]). See Fig. 5 for the comparison between different stimulation conditions and sessions.

\section{Relationship Between Training and Testing}

To investigate the relationship between performance in the training session and memory performance in the two testing sessions, we ran GEE analyses with the mean number of training blocks, the mean total exposure across all flags, and the mean total error across all flags as covariates. These analyses are summarised in Table 4 . The analyses showed a significant negative relationship between the covariate and performance in all three testing phases ( $p$ 's $\leq 0.005$ ), except for the relationship between mean total error and free recall that showed a non-significant effect $(p=0.101)$. These results show that whilst increased exposure leads to better memory recall within participants, those that required less training to achieve the performance criteria had improved long-term retention compared to those who required more training.

\section{Response Consistency}

To analyse the response consistency, non-parametric Spearman's correlation analysis was conducted between total exposure and total error. This analysis showed a significant correlation $(\mathrm{rs}(25)=0.704, p<0.001)$. Spearman's correlation analyses of performance in the three testing phases also showed significant results (free recall and cued recall: $\operatorname{rs}(25)=$ 
Table 3 Paired-samples $t$ tests comparing the two stimulation conditions over the two testing sessions

\begin{tabular}{lllllll}
\hline & $\beta$ & SE & $95 \%$ CI & Wald $\chi^{2}$ & $p$ & OR \\
\hline Follow-up 1 & & & & & & \\
$\quad$ Free recall & 0.020 & 0.155 & {$[-0.284,0.325]$} & 0.017 & 0.896 & 1.021 \\
$\quad$ Cued recall & 0.023 & 0.150 & {$[-0.270,0.316]$} & 0.024 & 0.878 & 1.023 \\
$\quad$ Timed cued recall & -2.493 & 1.974 & {$[-6.362,1.375]$} & 1.595 & 0.207 & \\
Follow-up 2 & & & & & & \\
Free recall & -0.211 & 0.143 & {$[-0.491,0.070]$} & 2.172 & 0.141 & 0.810 \\
Cued recall & -0.034 & 0.091 & {$[-0.213,0.145]$} & 0.139 & 0.709 & 0.967 \\
Timed cued recall & -5.080 & 1.174 & {$[-7.381,-2.779]$} & 18.728 & $<0.001 *$ & \\
\hline
\end{tabular}

$* p<0.008$ Bonferroni corrected for multiple comparison; $O R$, odd ratio; $S E$, standard error
$0.665, p<0.001$; free recall and timed cued recall: $\mathrm{rs}(25)=$ $0.580, p=0.002$; cued recall and timed cued recall: $\operatorname{rs}(25)=$ $0.759, p<0.001)$. The Spearman's correlations between mean training blocks and memory performance in the three testing phases showed significant negative correlations (free recall: rs $(25)=-0.654, p<0.001$; cued recall: $\operatorname{rs}(25)=-0.553, p=$ 0.004 ; timed cued recall: $\mathrm{rs}(25)=-0.836, p<0.001)$.

\section{Recency Effect}

Finally, to investigate the effect of recency on memory recall, GEE analyses were run with the number of the training session for each stimulus as covariate. These analyses are summarised in Table 5. These analyses showed a significant effect between the number of training sessions, as well as an interaction effect of session (follow-up 1 and 2) and training session. Therefore, to investigate the effect of training session on each testing session, we ran separate GEE analyses,
Table 6. These analyses showed an effect of recency in the first testing session (follow-up 1) only.

\section{Discussion}

Here, over a period of six training sessions, participants learnt 48 flags from around the world, whilst receiving either active or sham tDCS. They were then tested on these flags both 1 day and 1 week later. The results suggest many interesting findings. Firstly, contrary to previous literature showing potentially beneficial effects of tDCS on long- and short-term memory performance, we found that anodal tDCS over the left DLPFC did not improve learning ability compared to sham tDCS. Importantly, whilst no significant interaction was obtained between stimulation condition and session, due to our strong hypothesis, we ran post hoc analyses to investigate the effect of brain stimulation on performance over the two following sessions. These analyses indicated that participants performed significantly worse in
Table 4 Summary of the generalised estimating equation (GEE) investigating the relationship between performance in the training sessions and performance in the testing session

\begin{tabular}{llllll}
\hline & $\beta$ & SE & $95 \%$ CI & Wald $\chi^{2}$ & $p$ \\
\hline Mean training blocks & & & & & \\
Free recall & -1.256 & 0.302 & {$[-1.847,-0.664]$} & 17.307 & $<0.001^{*}$ \\
Cued recall & -1.999 & 0.704 & {$[-3.380,-0.619]$} & 8.057 & $0.005^{*}$ \\
$\quad$ Timed cued recall & -2.382 & 0.462 & {$[-3.287,-1.478]$} & 26.637 & $<0.001^{*}$ \\
Mean total exposure & & & & & \\
Free recall & -0.251 & 0.067 & {$[-0.382,-0.121]$} & 14.215 & $<0.001^{*}$ \\
Cued recall & -0.417 & 0.112 & {$[-0.636,-0.197]$} & 13.877 & $<0.001^{*}$ \\
Timed cued recall & -0.461 & 0.097 & {$[-0.651,-0.270]$} & 22.482 & $<0.001^{*}$ \\
Mean total error & & & & & \\
Free recall & -1.877 & 1.143 & {$[-4.117,0.363]$} & 2.697 & 0.101 \\
Cued recall & -7.102 & 1.815 & {$[-10.659,-3.546]$} & 15.320 & $<0.001^{*}$ \\
Timed cued recall & -7.239 & 1.367 & {$[-9.919,-4.559]$} & 28.028 & $<0.001^{*}$ \\
\hline
\end{tabular}

Mean training blocks refer to the mean of the number of required training blocks in the training sessions per participant. Mean total exposure refers to the mean of the number of presentations across all 48 flags per participant. Mean total error refers to the mean of the number of incorrect responses across all 48 flags per participant. * $p<0.008$ Bonferroni corrected for multiple comparison; $S E$, standard error 
Table 5 Summary of the generalised estimating equation (GEE) investigating the effect of recency on memory recall

\begin{tabular}{|c|c|c|c|c|c|c|}
\hline & $\beta$ & SE & $95 \% \mathrm{CI}$ & $\begin{array}{l}\text { Wald } \\
\chi^{2}\end{array}$ & $p$ & OR \\
\hline \multicolumn{7}{|l|}{ Free recall } \\
\hline Session & 1.417 & 0.310 & {$[0.810,2.025]$} & 20.911 & $<0.001 *$ & 4.126 \\
\hline Stimulation condition & 0.780 & 0.770 & {$[-0.729,2.290]$} & 1.027 & 0.311 & 2.182 \\
\hline Training session & 0.541 & 0.158 & {$[0.232,0.850]$} & 11.748 & $0.001 *$ & 1.718 \\
\hline Session $\times$ stimulation condition & -0.347 & 0.405 & {$[-1.142,0.447]$} & 0.734 & 0.392 & 0.707 \\
\hline Session $\times$ training session & -0.302 & 0.086 & {$[-0.471,-0.134]$} & 12.323 & $<0.001 *$ & 0.739 \\
\hline $\begin{array}{l}\text { Stimulation condition } \times \text { training } \\
\text { session }\end{array}$ & -0.288 & 0.214 & {$[-0.707,0.130]$} & 1.821 & 0.177 & 0.750 \\
\hline Three-way interaction & 0.163 & 0.109 & {$[-0.051,0.377]$} & 2.235 & 0.135 & 1.177 \\
\hline \multicolumn{7}{|l|}{ Cued recall } \\
\hline Session & 1.920 & 0.347 & {$[1.240,2.599]$} & 30.670 & $<0.001 *$ & 6.821 \\
\hline Stimulation condition & 0.506 & 0.599 & {$[-0.668,1.680]$} & 0.714 & 0.398 & 1.659 \\
\hline Training session & 0.569 & 0.135 & {$[0.304,0.834]$} & 17.664 & $<0.001 *$ & 1.766 \\
\hline Session $\times$ stimulation condition & -0.203 & 0.327 & {$[-0.843,0.437]$} & 0.387 & 0.534 & 0.816 \\
\hline Session $\times$ training session & -0.246 & 0.073 & {$[-0.390,-0.103]$} & 11.269 & $0.001 *$ & 0.782 \\
\hline $\begin{array}{l}\text { Stimulation condition } \times \text { training } \\
\text { session }\end{array}$ & -0.165 & 0.157 & {$[-0.472,0.141]$} & 1.117 & 0.291 & 0.848 \\
\hline Three-way interaction & 0.072 & 0.089 & {$[-0.101,0.246]$} & 0.669 & 0.413 & 1.075 \\
\hline
\end{tabular}

Training session indicates the number of the training session in which each stimulus was trained in. $O R$, odd ratio; $S E$, standard error the timed cued recall phase following active tDCS, than sham tDCS in the second testing session. Results also showed that participants had significantly better memory performance in free, cued, and timed cued recall phases in the second testing session (follow-up 2), compared to the first (follow-up 1), despite it being 1 week later with no exposure to flags. Finally, our analysis showed that memory performance was better for the flags that participants made more mistakes on, meaning they were also presented these flags more times. Further exploratory analyses showed that participants who performed better in the training sessions performed better in the testing sessions as well. Additionally, results showed a recency effect in the first testing session (follow-up 1), but not in the second testing session (follow-up 2).

Given that participants did not receive brain stimulation during the two testing sessions, perhaps superior performance in the sham tDCS condition could be attributed to a form of implicit state-dependent learning of tDCS: the state in the testing sessions being the same as the sham condition. Much care was taken to ensure the environmental context was kept the same between training and follow-up sessions; i.e. the task was carried out in the same room, by the same experimenter, with the same task and timings for the task, the only difference between the two was that instead of 8 flags being shown in the session; now, 48 flags were shown at once. There is much evidence to suggest that individuals can recall or recognise objects with greater accuracy when learning is performed in the same 'state' as the test phase. For example, it has been shown that when participants are asked to learn words whilst intoxicated by alcohol, they were able to recall significantly more of those words in the retrieval phase when intoxicated, compared to sober (Lowe, 1986; Petersen, 1977; Weingartner,
Table 6 Summary of the generalised estimating equation (GEE) analyses investigating the effect of recency on memory recall split between the two testing sessions (follow-up 1 and 2)

\begin{tabular}{rrrlcrl}
\hline & $\beta$ & SE & $95 \%$ CI & Wald $\chi^{2}$ & $p$ & OR \\
\hline Free recall & & & & & & \\
Session 1 & 0.175 & 0.046 & {$[0.085,0.265]$} & 14.592 & $<0.001^{*}$ & 1.191 \\
Session 2 & -0.042 & 0.038 & {$[-0.116,0.033]$} & 1.199 & 0.273 & 0.959 \\
Cued recall & & & & & \\
Session 1 & 0.275 & 0.042 & {$[0.193,0.358]$} & 42.649 & $<0.001 *$ & 1.317 \\
Session 2 & 0.066 & 0.030 & {$[0.008,0.124]$} & 4.902 & 0.027 & 1.068 \\
\hline
\end{tabular}

${ }^{*} p<0.012$ Bonferroni corrected for multiple comparison; $O R$, odd ratio; $S E$, standard error 
Adefris, Eich, \& Murphy, 1976; Weingartner \& Faillace, 1971). The same has also been shown with caffeine (Kelemen \& Creeley, 2003; Sanday et al., 2013) and nicotine (Peters \& McGee, 1982; Warburton, Wesnes, Shergold, \& James, 1986).

In the current study, the state dependency proposed is far more implicit than both environmental and pharmacological state dependency, as participants would have almost certainly not perceived any physiological changes, aside from an initial itching sensation at the site of stimulation. For transcranial alternating current stimulation (tACS), it has been shown that by encouraging certain oscillatory activity during the encoding phase, more accurate results are obtained if the same pattern is reinstated during retrieval, compared to a different frequency (Javadi, Glen, Halkiopoulos, Schulz, \& Spiers, 2017). Although this is not directly comparable to tDCS, it highlights that brain stimulation can potentially produce a form of state dependent learning. Whilst it has been shown that tDCS can evoke neuronal changes in the brain (Nitsche \& Paulus, 2000), it is not understood whether an absence of these specific changes in a later test phase, without the presence of stimulation, can interfere with recall and recognition performance. Therefore, future research should look to address whether a potential state dependency is present for tDCS, in particular, when extended retention intervals are used.

Another reason for this discrepancy in findings may be due in part to varying behavioural tasks used to assess the effectiveness of tDCS in previous literature. When looking at similar studies that have administered anodal tDCS over the left DLPFC during encoding, but not retrieval, LTM performances have shown an increase following stimulation (Javadi, Cheng, \& Walsh, 2012; Javadi \& Walsh, 2012). Previous studies investigating the effects of tDCS on LTM have often used less cognitively demanding behavioural tasks, such as old/new recognition tasks (Javadi \& Cheng, 2013; Javadi et al., 2012; Javadi \& Walsh, 2012), or pairedassociate learning tasks, where they must recall the word originally shown to pair another word (de Lara et al., 2017; Garside, Arizpe, Lau, Goh, \& Walsh, 2015). The behavioural task used here has a greater degree of complexity. Whilst comparable to a paired-associate learning task, in that two stimuli are paired (e.g. a picture and a word), it also requires the participant to instantly retrieve this association under time pressure. The extra degree of complexity involved in this task may be above and beyond the abilities that tDCS has to modulate performance. Therefore, this may be one reason why no effect of stimulation was found during training.

Interestingly, a recent study has shown anodal tDCS to be of detriment to verbal LTM. Using a traditional retrieval-based learning task, Brunyé et al. (2018) found anodal tDCS to have no effect on immediate verbal learning. In a subsequent recall test 2 days later, verbal LTM was actually impaired following anodal tDCS to the left DLPFC. One theory proposed by Miniussi, Harris and Ruzzoli (2013), states that whilst anodal tDCS can facilitate improvements for well-trained or familiar tasks, it is ineffective for novel tasks. This is because when learning a new task, neurons initially fire unsystematically until consolidation of the task has occurred. Based on evidence gathered from anodal tDCS of the motor cortex, anodal tDCS induces membrane depolarisation for neurons around the target area (Nitsche et al., 2008); the stimulation adds to the neuronal noise around the site; thus, a clear signal cannot emerge. Furthermore, a meta-analysis by Jacobson, Koslowsky and Lavidor (2012) found tDCS to modulate performance for procedural tasks, but rarely cognitive tasks, claiming that cognitive functions are typically supported by richer brain networks, far more complex than motor areas. They also stated that cognitive experiments yield far more heterogeneous results, adding controversy to the growing body of literature surrounding tDCS and LTM, but also highlighting the complex nature of LTM mechanisms. It could be suggested that learning via retrieval, an already complex notion, may be reliant on a number of cognitive domains/richer brain networks, and therefore, an intervention such as tDCS may lack the power to modulate such composite procedures.

Another interesting finding is that participants recognised a significantly greater number of flags in the second testing session (follow-up 2), 1 week later with no exposure to flags, compared to the first test session. This was the case for both cued and free recall. It is traditionally understood that declarative memory degrades with passage of time. Studies comparing immediate with delayed tests following lengthy retention intervals have often seen a decline, or at best maintenance of memory performance (Karpicke \& Roediger, 2007; Roediger \& Karpicke, 2006). However, improvements in performance following training are well represented for non-declarative memory (Walker, Brakefield, Morgan, Hobson, \& Stickgold, 2002; Walker et al., 2003; Walker \& Stickgold, 2004). These studies showed improvements in performance of procedural tasks following periods of sleep, compared to identical periods of wakefulness. Whilst the retention intervals in these studies are shorter, they highlight the value of sleep for procedural memory consolidation. Perhaps the retrieval-based learning task adopted in this study should be considered to encompass more than just declarative memory. Therefore, sleep, as with motor sequence tasks, may facilitate a delayed improvement.

When looking at how performance in the training phase affected subsequent performance in the test phases, interesting results were obtained. Firstly, the results suggested that flags that participants frequently got wrong were more likely to be 
remembered during the testing phases. This is perhaps to be expected as an incorrect response also means that feedback for that particular flag is provided more times and the flag is presented more frequently also. From this, it may be assumed that learning was facilitated in two ways: errorful learning and repeated exposure. Errorful learning (guessing incorrectly) has been shown to enhance subsequent memory performance, only when a study opportunity, in this case, feedback, is presented immediately afterwards (Knight, Hunter Ball, Brewer, DeWitt, \& Marsh, 2012; Kornell, Hays, \& Bjork, 2009; Vaughn \& Rawson, 2012). Whilst there is still some debate about the effectiveness of errorful learning, the increased exposure that accompanies error making in this experiment will no doubt have affected subsequent retention.

The final point of discussion obtained from the results is a recency effect, only observable in the first testing session. As the participants learnt each group of flags on consecutive days, it is to be expected that a recency effect would be observed. One of the objectives of the self-paced cued recall was to provide feedback to wash out any recency effects for the timed cued recall. This, as well as the 1-week retention interval, removed the recency effect for the second testing session. This time delay has been shown to reduce recency effects in several instances (Glenberg \& Kraus, 1981; Talmi \& GoshenGottstein, 2006; Toppino \& Bloom, 2002).

\section{Considerations and Directions for Future Research}

There are several methodological considerations that should be discussed following the interpretation of these results. Firstly, as the study adopted a pseudo-randomised stimulation pattern, there were instances where a sham protocol would have followed a stimulation protocol the previous day. There is some debate to the temporal curve of stimulation and its effects after stimulation have been completed. It has been shown that stimulation effects can last up to $90 \mathrm{~min}$ after stimulation has ended (Nitsche \& Paulus, 2000). However, there is evidence to suggest this may be much longer, depending on the duration and intensity of the intervention (Manenti et al., 2015; Marangolo et al., 2013). It is therefore important to acknowledge that, due to the procedure of this study, carry over effects may have had some impact on sham stimulation.

The mixed stimulation protocol and nature of the task used in this study also meant that depending on performance, some participants would have completed the task during stimulation (online), with others completing it after cessation of stimulation (offline). Whilst this is an important consideration, the authors believe that, due to the literature highlighting negligible differences between online and offline protocols (Hill et al., 2016; Oldrati, Colombo, \& Antonietti, 2018; Pirulli, Fertonani, \& Miniussi, 2013), as well as the fact that stimulation effects can last more than an hour after cessation of stimulation (Nitsche \& Paulus, 2000), this will have had little impact on the results. Nevertheless, this is an important aspect of the protocol to consider.

It is also important to point out that the cued recall phase consisted of the presentation of all the flags followed by feedback. This was to give the chance to participants to recall the flags from their long-term memory and test their reaction time in the time cued recall phase. One consideration of this may be that the delayed improvement found in the second testing session may be attributed to an extra exposure of flags and the provided feedback. Additionally, inferior performance in the first testing session may be due to the novelty of the task. Improved results in the second testing session may be attributed to an increase in task familiarity, in particular, for the free recall and cued recall phases. Therefore, to study the exact underlying mechanism of memory improvement, future studies should have groups with different retention intervals without multiple testing sessions.

Based upon the theory proposed by Miniussi et al. (2013), which claims that tDCS is only effective for familiar tasks, perhaps the lack of difference between active and sham conditions is not unexpected. Particularly, this task requires participants to respond under timed pressure. If the participants received additional training prior to the administration of stimulation, familiarising them with the task, perhaps stimulation may have been more successful. Future research could address this by using more familiar tasks or increase the number of training sessions.

Finally, conventional tDCS is not a very focal stimulation method. As shown in Fig. 3, brain areas other than the left DLPFC were stimulated concurrently, such as the anterior part of left superior temporal gyrus and Broca's area, regions associated with language such as the semantic meaning of objects and speech fluency (Hinke et al., 1993; Leff et al., 2009; Visser \& Lambon Ralph, 2011). Therefore, although not significant, there is a possibility that participants achieved the learning performance threshold in the active tDCS condition faster than the sham tDCS condition, leading to inferior memory performance at later stages. Therefore, future research should design studies that are free of such bias.

\section{Conclusion}

Using a variation of a retrieval-based learning task, we have shown that anodal tDCS to the left DLPFC has no effect on learning ability and is of detriment to subsequent retrieval of flag-country association. The SAFMEDS task used here requires a great degree of executive functioning, which is common in daily life. Therefore, the results obtained here serve to highlight the inconsistencies associated with tDCS for practical implications. It also leads us to ask whether this particular task can enhance consolidation, only evident after a long 
retention interval. The SAFMEDS task has deeply entrenched roots in ABA, with many applications used for individuals with learning difficulties. By gaining a greater understanding of how this task works from a neuroscience perspective, its benefits cannot only be seen from an educational perspective but could also extend into neuro-rehabilitative domains.

Acknowledgements The authors would like to thank Necati Kartal for his help in piloting the study.

Data Availability Associated data can be downloaded from https://doi. org/10.17605/OSF.IO/7RZFJ

\section{Compliance with Ethical Standards}

Written informed consent was received from all participants before the study began. The procedure of the study was approved by the local ethics committee in the School of Psychology at the University of Kent. All procedures were in accordance with the 1964 Helsinki declaration and its later amendments.

Conflict of Interest The authors declare that they have no conflict of interest.

\section{Appendix}

Table 7 List of the 60 flags used in the baseline session. Forty-eight of these flags that were unknown to each participant were subsequently chosen at random for the training sessions

\begin{tabular}{|c|c|c|c|c|c|}
\hline Afghanistan & China & Guatemala & Kyrgyzstan & Nepal & Suriname \\
\hline Albania & Colombia & Guinea & Laos & Nigeria & Swaziland \\
\hline Algeria & Comoros & Haiti & Lebanon & Panama & Tanzania \\
\hline Angola & Croatia & Honduras & Lesotho & Peru & Thailand \\
\hline Barbados & Cuba & Iceland & Libya & Philippines & Togo \\
\hline Belize & Egypt & India & Liechtenstein & Qatar & Tunisia \\
\hline Bhutan & Estonia & Indonesia & Madagascar & Rwanda & Uganda \\
\hline Botswana & Gabon & Japan & Malta & Seychelles & Vanuatu \\
\hline Brunei & Georgia & Kazakhstan & Mexico & Somalia & Zambia \\
\hline Cameroon & Grenada & Kenya & Morocco & Sudan & Zimbabwe \\
\hline
\end{tabular}

Open Access This article is licensed under a Creative Commons Attribution 4.0 International License, which permits use, sharing, adaptation, distribution and reproduction in any medium or format, as long as you give appropriate credit to the original author(s) and the source, provide a link to the Creative Commons licence, and indicate if changes were made. The images or other third party material in this article are included in the article's Creative Commons licence, unless indicated otherwise in a credit line to the material. If material is not included in the article's Creative Commons licence and your intended use is not permitted by statutory regulation or exceeds the permitted use, you will need to obtain permission directly from the copyright holder. To view a copy of this licence, visit http://creativecommons.org/licenses/by/4.0/.

\section{References}

Accornero, N., Capozza, M., Pieroni, L., Pro, S., Davì, L., \& Mecarelli, O. (2014). EEG mean frequency changes in healthy subjects during prefrontal transcranial direct current stimulation. Journal of Neurophysiology, 112(6), 1367-1375. https://doi.org/10.1152/jn. 00088.2014.

Agarwal, P. K., Karpicke, J. D., Kang, S. H. K., Roediger, H. L., \& McDermott, K. B. (2008). Examining the testing effect with openand closed-book tests. Applied Cognitive Psychology, 22(7), 861876. https://doi.org/10.1002/acp.1391.
Andrews, S. C., Hoy, K. E., Enticott, P. G., Daskalakis, Z. J., \& Fitzgerald, P. B. (2011). Improving working memory: the effect of combining cognitive activity and anodal transcranial direct current stimulation to the left dorsolateral prefrontal cortex. Brain Stimulation, 4(2), 84-89. https://doi.org/10.1016/j.brs.2010.06.004.

Antal, A., Nitsche, M. A., Kincses, T. Z., Kruse, W., Hoffmann, K. P., \& Paulus, W. (2004). Facilitation of visuo-motor learning by transcranial direct current stimulation of the motor and extrastriate visual areas in humans. European Journal of Neuroscience, 19(10), 28882892. https://doi.org/10.1111/j.1460-9568.2004.03367.x.

Au, J., Karsten, C., Buschkuehl, M., \& Jaeggi, S. M. (2017). Optimizing transcranial direct current stimulation protocols to promote longterm learning. Journal of Cognitive Enhancement, 1(1), 65-72. https://doi.org/10.1007/s41465-017-0007-6.

Bestmann, S., de Berker, A. O., \& Bonaiuto, J. (2015). Understanding the behavioural consequences of noninvasive brain stimulation. Trends in Cognitive Sciences, 19(1), 13-20. https://doi.org/10.1016/j.tics. 2014.10.003.

Brunoni, A. R., \& Vanderhasselt, M.-A. (2014). Working memory improvement with non-invasive brain stimulation of the dorsolateral prefrontal cortex: a systematic review and meta-analysis. Brain and Cognition, 86, 1-9. https://doi.org/10.1016/j.bandc.2014.01.008.

Brunyé, T. T., Smith, A. M., Horner, C. B., \& Thomas, A. K. (2018). Verbal long-term memory is enhanced by retrieval practice but impaired by prefrontal direct current stimulation. Brain and Cognition, 128, 80-88. https://doi.org/10.1016/j.bandc.2018.09.008. 
Coffman, B. A., Clark, V. P., \& Parasuraman, R. (2014). Battery powered thought: enhancement of attention, learning, and memory in healthy adults using transcranial direct current stimulation. NeuroImage, 85 , 895-908. https://doi.org/10.1016/j.neuroimage.2013.07.083.

de Lara, G. A., Knechtges, P. N., Paulus, W., \& Antal, A. (2017). Anodal tDCS over the left DLPFC did not affect the encoding and retrieval of verbal declarative information. Frontiers in Neuroscience, 11(AUG), 1-9. https://doi.org/10.3389/fnins.2017.00452.

Dedoncker, J., Brunoni, A. R., Baeken, C., \& Vanderhasselt, M.-A. (2016). A systematic review and meta-analysis of the effects of transcranial direct current stimulation (tDCS) over the dorsolateral prefrontal cortex in healthy and neuropsychiatric samples: influence of stimulation parameters. Brain Stimulation, 9(4), 501-517. https:// doi.org/10.1016/j.brs.2016.04.006.

Fabrizio, M. A., \& Moors, A. L. (2017). Evaluating mastery: measuring instructional outcomes for children with autism. European Journal of Behavior Analysis, 4(1-2), 23-36. https://doi.org/10.1080/ 15021149.2003.11434213.

Fazio, L. K., \& Marsh, E. J. (2019). Retrieval-based learning in children. Current Directions in Psychological Science, 28(2), 111-116. https://doi.org/10.1177/0963721418806673.

Fertonani, A., \& Miniussi, C. (2017). Transcranial electrical stimulation: what we know and do not know about mechanisms. Neuroscientist, 23(2), 109-123. https://doi.org/10.1177/1073858416631966.

Fregni, F., Boggio, P. S., Nitsche, M., Bermpohl, F., Antal, A., Feredoes, E., et al. (2005). Anodal transcranial direct current stimulation of prefrontal cortex enhances working memory. Experimental Brain Research, 166(1), 23-30. https://doi.org/10.1007/s00221-0052334-6.

Garside, P., Arizpe, J., Lau, C. I., Goh, C., \& Walsh, V. (2015). Cross-hemispheric alternating current stimulation during a nap disrupts slow wave activity and associated memory consolidation. Brain Stimulation, 8(3), 520-527. https://doi.org/ 10.1016/j.brs.2014.12.010.

Glenberg, A. M., \& Kraus, T. A. (1981). Long-term recency is not found on a recognition test. Journal of Experimental Psychology: Human Learning and Memory, 7(6), 475-479. https://doi.org/10.1037/ 0278-7393.7.6.475.

Graf, S. A., \& Auman, J. (2005). SAFMEDS : a tool to build fluency.

Gray, S. J., Brookshire, G., Casasanto, D., \& Gallo, D. A. (2015). Electrically stimulating prefrontal cortex at retrieval improves recollection accuracy. Cortex, 73, 188-194. https://doi.org/10.1016/j. cortex.2015.09.003.

Hecht, D., Walsh, V., \& Lavidor, M. (2010). Transcranial direct current stimulation facilitates decision making in a probabilistic guessing task. Journal of Neuroscience, 30(12), 4241-4245. https://doi.org/ 10.1523/jneurosci.2924-09.2010.

Hill, A. T., Fitzgerald, P. B., \& Hoy, K. E. (2016). Effects of anodal transcranial direct current stimulation on working memory: a systematic review and meta-analysis of findings from healthy and neuropsychiatric populations. Brain Stimulation, 9(2), 197-208. https:// doi.org/10.1016/j.brs.2015.10.006

Hinke, R. M., Hu, X., Stillman, A. E., Kim, S. G., Merkle, H., Salmi, R., \& Ugurbil, K. (1993). Functional magnetic resonance imaging of brocas area during internal speech. NeuroReport, 4(6), 675-678. https://doi.org/10.1097/00001756-199306000-00018.

Homan, R., Herman, J., \& Purdy, P. (1987). Cerebral location of international 10-20 system electrode placement. Electroencephalography and Clinical Neurophysiology, 4(66), 376.

Horvath, J. C., Forte, J. D., \& Carter, O. (2015a). Quantitative review finds no evidence of cognitive effects in healthy populations from single-session transcranial direct current stimulation (tDCS). Brain Stimulation, 8(3), 535-550. https://doi. org/10.1016/j.brs.2015.01.400.

Horvath, J. C., Forte, J. D., \& Carter, O. (2015b). Evidence that transcranial direct current stimulation (tDCS) generates little-to-no reliable neurophysiologic effect beyond MEP amplitude modulation in healthy human subjects: a systematic review. Neuropsychologia, 66(36) Elsevier Ltd., 213. https://doi.org/10.1016/j. neuropsychologia.2014.11.021.

Huang, Y., Datta, A., Bikson, M., \& Parra, L. C. (2019). Realistic volumetric-approach to simulate transcranial electric stimulationROAST - a fully automated open-source pipeline. Journal of Neural Engineering, 16(5), 056006.

Jacobson, L., Koslowsky, M., \& Lavidor, M. (2012). tDCS polarity effects in motor and cognitive domains: a meta-analytical review. Experimental Brain Research, 216(1), 1-10. https://doi.org/10. 1007/s00221-011-2891-9.

Jarosz, A. F., \& Wiley, J. (2014). What are the odds? A practical guide to computing and reporting Bayes factors. The Journal of Problem Solving, 7(1), 2.

Javadi, A.-H., \& Cheng, P. (2013). Transcranial direct current stimulation (tDCS) enhances reconsolidation of long-term memory. Brain Stimulation, 6(4), 668-674. https://doi.org/ 10.1016/j.brs.2012.10.007.

Javadi, A.-H., \& Walsh, V. (2012). Transcranial direct current stimulation (tDCS) of the left dorsolateral prefrontal cortex modulates declarative memory. Brain Stimulation, 5(3), 231-241. https://doi.org/10. 1016/j.brs.2011.06.007.

Javadi, A.-H., Cheng, P., \& Walsh, V. (2012). Short duration transcranial direct current stimulation (tDCS) modulates verbal memory. Brain Stimulation, 5(4), 468-474. https://doi.org/10.1016/j.brs.2011.08. 003.

Javadi, A.-H., Brunec, I. K., Walsh, V., Penny, W. D., \& Spiers, H. J. (2014). Transcranial electrical brain stimulation modulates neuronal tuning curves in perception of numerosity and duration. NeuroImage, 102, 451-457. https://doi.org/10.1016/j.neuroimage. 2014.08.016.

Javadi, A.-H., Glen, J. C., Halkiopoulos, S., Schulz, M., \& Spiers, H. J. (2017). Oscillatory reinstatement enhances declarative memory. The Journal of Neuroscience, 37(41), 9939-9944. https://doi.org/10. 1523/JNEUROSCI.0265-17.2017.

Karpicke, J. D., \& Aue, W. R. (2015). The testing effect is alive and well with complex materials. Educational Psychology Review, 27(2), 317-326. https://doi.org/10.1007/s10648-015-9309-3.

Karpicke, J. D., \& Grimaldi, P. J. (2012). Retrieval-based learning: a perspective for enhancing meaningful learning. Educational Psychology Review, 24(3), 401-418. https://doi.org/10.1007/ s10648-012-9202-2.

Karpicke, J. D., \& Roediger, H. L. (2007). Repeated retrieval during learning is the key to long-term retention. Journal of Memory and Language, 57(2), 151-162. https://doi.org/10.1016/j.jml.2006.09. 004.

Karpicke, J. D., \& Roediger, H. L. (2008). The critical importance of retrieval for learning. Science, 319(5865), 966-968. https://doi.org/ 10.1126/science. 1152408 .

Karpicke, J. D., Lehman, M., \& Aue, W. R. (2014). Chapter seven Retrieval-based learning: An episodic context account. In: B. H. Ross (Ed.), Psychology of learning and motivation (Vol. 61, pp. 237-284). Academic Press Inc. https://doi.org/10.1016/B978-0-12800283-4.00007-1.

Katz, B., Au, J., Buschkuehl, M., Abagis, T., Zabel, C., Jaeggi, S. M., \& Jonides, J. (2017). Individual differences and long-term consequences of tDCS-augmented cognitive training. Journal of Cognitive Neuroscience, 29(9), 1498-1508. https://doi.org/10. 1162/jocn_a_01115.

Kelemen, W. L., \& Creeley, C. E. (2003). State-dependent memory effects using caffeine and placebo do not extend to metamemory. The Journal of General Psychology, 130(1), 70-86. https://doi.org/10. 1080/00221300309601276.

Knight, J. B., Hunter Ball, B., Brewer, G. A., DeWitt, M. R., \& Marsh, R. L. (2012). Testing unsuccessfully: a specification of the underlying 
mechanisms supporting its influence on retention. Journal of Memory and Language, 66(4), 731-746. https://doi.org/10.1016/j. jml.2011.12.008.

Kornell, N., Hays, M. J., \& Bjork, R. A. (2009). Unsuccessful retrieval attempts enhance subsequent learning. Journal of Experimental Psychology. Learning, Memory, and Cognition, 35(4), 989-998. https://doi.org/10.1037/a0015729.

Leff, A. P., Schofield, T. M., Crinion, J. T., Seghier, M. L., Grogan, A., Green, D. W., \& Price, C. J. (2009). The left superior temporal gyrus is a shared substrate for auditory short-term memory and speech comprehension: evidence from 210 patients with stroke. Brain, 132(12), 3401-3410. https://doi.org/10.1093/brain/awp273.

Leshikar, E. D., Leach, R. C., McCurdy, M. P., Trumbo, M. C., Sklenar, A. M., Frankenstein, A. N., \& Matzen, L. E. (2017). Transcranial direct current stimulation of dorsolateral prefrontal cortex during encoding improves recall but not recognition memory. Neuropsychologia, 106(September), 390-397. https://doi.org/10. 1016/j.neuropsychologia.2017.10.022.

Loftus, A. M., Yalcin, O., Baughman, F. D., Vanman, E. J., \& Hagger, M. S. (2015). The impact of transcranial direct current stimulation on inhibitory control in young adults. Brain and Behavior: A Cognitive Neuroscience Perspective, 5(5), e00332. https://doi.org/10.1002/ brb3.332.

Lowe, G. (1986). State-dependent learning effects with a combination of alcohol and nicotine. Psychopharmacology., 89(1), 105-107. https://doi.org/10.1007/BF00175199.

Manenti, R., Brambilla, M., Petesi, M., Ferrari, C., \& Cotelli, M. (2013). Enhancing verbal episodic memory in older and young subjects after non-invasive brain stimulation. Frontiers in Aging Neuroscience, 5(SEP), 49. https://doi.org/10.3389/fnagi.2013.00049.

Manenti, R., Petesi, M., Brambilla, M., Rosini, S., Miozzo, A., Padovani, A., et al. (2015). Efficacy of semantic-phonological treatment combined with tDCS for verb retrieval in a patient with aphasia. Neurocase, 21(1), 109-119. https://doi.org/10.1080/13554794. 2013.873062 .

Marangolo, P., Fiori, V., Di Paola, M., Cipollari, S., Razzano, C., Oliveri, M., \& Caltagirone, C. (2013). Differential involvement of the left frontal and temporal regions in verb naming: a tDCS treatment study. Restorative Neurology and Neuroscience, 31(1), 63-72. https://doi.org/10.3233/RNN-120268.

McDaniel, M. A., \& Masson, M. E. J. (1985). Altering memory representations through retrieval. Journal of Experimental Psychology: Learning, Memory, and Cognition, 11(2), 371-385. https://doi.org/ 10.1037/0278-7393.11.2.371.

Michael, J. (1993). Establishing operations. The Behavior Analyst, 16(2), 191-206. https://doi.org/10.1007/BF03392623.

Miniussi, C., Harris, J. A., \& Ruzzoli, M. (2013, September). Modelling non-invasive brain stimulation in cognitive neuroscience. Neuroscience and Biobehavioral Reviews, 37(8), 1702-1712. https://doi.org/10.1016/j.neubiorev.2013.06.014.

Nasseri, P., Nitsche, M. A., \& Ekhtiari, H. (2015). A framework for categorizing electrode montages in transcranial direct current stimulation. Frontiers in Human Neuroscience, 9, 54. https://doi.org/10. 3389/fnhum.2015.00054.

Nitsche, M. A., \& Paulus, W. (2000). Excitability changes induced in the human motor cortex by weak transcranial direct current stimulation. The Journal of Physiology, 527(3), 633-639. https://doi.org/10. 1111/j.1469-7793.2000.t01-1-00633.x.

Nitsche, M. A., Schauenburg, A., Lang, N., Liebetanz, D., Exner, C., Paulus, W., \& Tergau, F. (2003). Facilitation of implicit motor learning by weak transcranial direct current stimulation of the primary motor cortex in the human. Journal of Cognitive Neuroscience, 15(4), 619-626. https://doi.org/10.1162/089892903321662994.

Nitsche, M., Cohen, L. G., Wassermann, E. M., Priori, A., Lang, N., Antal, A., et al. (2008). Transcranial direct current stimulation: state of the art 2008. Brain Stimulation, 1(3), 206-223. https://doi.org/10. 1016/j.brs.2008.06.004.

Oldrati, V., Colombo, B., \& Antonietti, A. (2018). Combination of a short cognitive training and tDCS to enhance visuospatial skills: a comparison between online and offline neuromodulation. Brain Research, 1678, 32-39. https://doi.org/10.1016/j.brainres.2017.10. 002.

Olson, R., Laraway, S., \& Austin, J. (2001). Unconditioned and conditioned establishing operations in organizational behavior management. Journal of Organizational Behavior Management, 21(2), 7 35. https://doi.org/10.1300/J075v21n02_03.

Parkin, B. L., Ekhtiari, H., \& Walsh, V. F. (2015). Non-invasive human brain stimulation in cognitive neuroscience: a primer. Neuron, 87(5), 932-945. https://doi.org/10.1016/j.neuron.2015.07.032.

Peters, R., \& McGee, R. (1982). Cigarette smoking and state-dependent memory. Psychopharmacology., 76, 232-235. https://doi.org/10. 1007/BF00432551.

Petersen, R. C. (1977). Retrieval failures in alcohol state-dependent learning. Psychopharmacology, 55(2), 141-146. https://doi.org/10.1007/ BF01457849.

Philiastides, M. G., Auksztulewicz, R., Heekeren, H. R., \& Blankenburg, F. (2011). Causal role of dorsolateral prefrontal cortex in human perceptual decision making. Current Biology, 21(11), 980-983. https://doi.org/10.1016/j.cub.2011.04.034.

Pirulli, C., Fertonani, A., \& Miniussi, C. (2013). The role of timing in the induction of neuromodulation in perceptual learning by transcranial electric stimulation. Brain Stimulation, 6(4), 683-689. https://doi. org/10.1016/j.brs.2012.12.005.

Roediger, H. L., \& Butler, A. C. (2011). The critical role of retrieval practice in long-term retention. Trends in Cognitive Sciences, 15(1), 20-27. https://doi.org/10.1016/j.tics.2010.09.003.

Roediger, H. L., \& Karpicke, J. D. (2006). Test-enhanced learning: taking memory tests improves long-term retention. Psychological Science, 17(3), 249-255. https://doi.org/10.1111/j.1467-9280.2006.01693.x.

Rosen, V. M., \& Engle, R. W. (1997). The role of working memory capacity in retrieval. Journal of Experimental Psychology: General, 126(3), 211-227. https://doi.org/10.1037/0096-3445.126. 3.211.

Sanday, L., Zanin, K. A., Patti, C. L., Fernandes-Santos, L., Oliveira, L. C., Longo, B. M., et al. (2013). Role of state-dependent learning in the cognitive effects of caffeine in mice. International Journal of Neuropsychopharmacology, 16(7), 1547-1557. https://doi.org/10. 1017/S1461145712001551.

Sara, S. J. (2000). Retrieval and reconsolidation: toward a neurobiology of remembering. Learning and Memory, 7(2), 73-84. https://doi. org/10.1101/lm.7.2.73.

Sundberg, M. L. (1993). The application of establishing operations. The Behavior Analyst, 16(2), 211-214. https://doi.org/10.1007/ BF03392625.

Talmi, D., \& Goshen-Gottstein, Y. (2006). The long-term recency effect in recognition memory. Memory, 14(4), 424-436. https://doi.org/10. 1080/09658210500426623.

Toppino, T. C., \& Bloom, L. C. (2002). The spacing effect, free recall, and two-process theory: a closer look. Journal of Experimental Psychology. Learning, Memory, and Cognition, 28(3), 437-444. https://doi.org/10.1037/0278-7393.28.3.437.

Vaughn, K. E., \& Rawson, K. A. (2012). When is guessing incorrectly better than studying for enhancing memory? Psychonomic Bulletin and Review, 19(5), 899-905. https://doi.org/10.3758/s13423-0120276-0.

Visser, M., \& Lambon Ralph, M. A. (2011). Differential contributions of bilateral ventral anterior temporal lobe and left anterior superior temporal gyrus to semantic processes. Journal of Cognitive Neuroscience, 23(10), 3121-3131. https://doi.org/10.1162/jocn a 00007. 
Walker, M. P., \& Stickgold, R. (2004). Sleep-dependent learning and memory consolidation. Neuron, 44(1), 121-133. https://doi.org/10. 1016/j.neuron.2004.08.031.

Walker, M. P., Brakefield, T., Morgan, A., Hobson, J. A., \& Stickgold, R. (2002). Practice with sleep makes perfect: sleep-dependent motor skill learning. Neuron, 35(1), 205-211. https://doi.org/10.1016/ S0896-6273(02)00746-8.

Walker, M. P., Brakefield, T., Seidman, J., Morgan, A., Hobson, J. A., \& Stickgold, R. (2003). Sleep and the time course of motor skill learning. Learning and Memory, 10(4), 275-284. https://doi.org/10. 1101/lm.58503.

Warburton, D., Wesnes, K., Shergold, K., \& James, M. (1986). Facilitation of learning and state dependency with nicotine. Psychopharmacology, 89(1), 55-59. https://doi.org/10.1007/ BF00175189.

Weingartner, H., \& Faillace, L. (1971). Alcohol state-dependent learning in man. The Journal of Nervous and Mental Disease, 153(6), 395406. https://doi.org/10.1097/00005053-197112000-00003.
Weingartner, H., Adefris, W., Eich, J. E., \& Murphy, D. L. (1976). Encoding-imagery specificity in alcohol state-dependent learning. Journal of Experimental Psychology: Human Learning and Memory, 2(1), 83-87. https://doi.org/10.1037/0278-7393.2.1.83.

Zaehle, T., Sandmann, P., Thorne, J. D., Jäncke, L., \& Herrmann, C. S. (2011). Transcranial direct current stimulation of the prefrontal cortex modulates working memory performance: combined behavioural and electrophysiological evidence. BMC Neuroscience, 12(1), 2. https://doi.org/10.1186/1471-2202-12-2.

Zayac, R. M., \& Johnston, J. M. (2008). Contriving establishing operations: responses of individuals with developmental disabilities during a learning task. Research in Developmental Disabilities, 29(3), 202-216. https://doi.org/10.1016/j.ridd.2007.03.001.

Publisher's Note Springer Nature remains neutral with regard to jurisdictional claims in published maps and institutional affiliations. 